\title{
Modeling the rheological properties of highly nano-filled polymers
}

\author{
Hajir Kourki ', Mehrzad Mortezaei ${ }^{2}$, \\ Mohammad Hossein Navid Famili ${ }^{3}$ and Milad Malekipirbazari ${ }^{4}$
}

\begin{abstract}
Organic and inorganic materials are usually added to polymers in order to achieve some benefits such as reducing the product cost, as well as achieving higher modulus and strength. Addition of these materials would change polymers' behavior. Adding nano-materials to polymers on the other hand is a new challenge in the field of polymer composites where previous studies were unable to achieve good correlation with nano-composites at higher particle volume fractions. In this research, Yamamoto network theory is developed to investigate the behavior of highly nano-filled systems. For this purpose, five different types of sub-chain and two types of junctions are considered and the effect of particle size, concentration, and the model parameters in association with the behavior of the junctions are studied. Moreover, some experiments are performed on polystyrene filled with nano-silica at different particle size and concentration values in frequency mod in the linear region. At last, we compared the results of our final model with the experiments in order to evaluate its accuracy, which confirmed a very good agreement.
\end{abstract}

\section{Keywords}

Rheology, nanocomposites, modeling, network theory, particle size and particle concentration

\section{Introduction}

Predicting the properties of polymers is one of the major concerns. In this regard, various models have been developed to predict the properties of polymers in the solution or melt states, among which Rouse ${ }^{1}$ proposed a theory for the dilute solution of coiling polymers. In this model, thermodynamic potential energy is the major term, which changes with motion in the system. Tracking the motion and variations of the potential energy leads to the prediction of solution behavior.

Besides the dilute solution, the behavior of concentrate solution or polymer melts is also a major concern. Yamomoto $^{2-4}$ considered polymer melts as a network with temporary junction, where the spatial distribution of these junctions determines the properties of the polymer systems. Theory of Reptation presents another interpretation of polymer chains movement in the melt state or the concentrated solution. De Gennes ${ }^{5}$ employed this theory in order to predict the relaxation time of the polymer chains. Based on De Gennes' model, a defect on the polymer chains, which is caused by deformation on that, can diffuse along the polymer backbone and relax deformed chain to the equilibrium state. Doi and
Edward ${ }^{6}$ defined a random process based on Reptation theory concerning the movement of polymer chains, as well as estimated relaxation time and modulus for the polymer melts and concentration solution.

In addition to the polymer solution or melts, existence of filler particles in polymers would change their properties. The hydrodynamic motion of these particles is their first effect on the flow behavior of filled systems. This effect is described as Einstein's equation ${ }^{7}$ and was proved by Advani. ${ }^{8}$ Polymer molecules interact with the surface of particles. ${ }^{9}$ This interaction is because of

\footnotetext{
'Polymer Engineering Group, Chemical Engineering Department, Graduate University of Advanced Technology, Iran

${ }^{2}$ Polymer Engineering Group, Composite Science and Technology Research Center, Iran

${ }^{3}$ Faculty of Chemical Engineering, Polymer Engineering Department, Tarbiat Modares University, Iran

${ }^{4}$ Department of Industrial Engineering, Bilkent University, Ankara, Turkey

\section{Corresponding author:}

Hajir Kourki, Polymer Engineering Group, Chemical Engineering Department, Graduate University of Advanced Technology, Kerman, Iran. Email: hajir.kourki@kgut.ac.ir
} 
hydrogen bonding and leads to polymer adsorption on the particle surfaces. ${ }^{10}$ Doremus and Piau assumed that the adsorbed polymer creates a network parallel to the free polymers, and estimated the Yamamoto model parameters for the double network. ${ }^{11}$ They tried to represent the yielding behavior of the filled system with the double network theory. Parameters of this theory with the molecular perspective were described by Sarvestani, ${ }^{12}$ where polymer molecules were divided into bridge, dangle and loop sub-chains, and the network parameters for every sub-chain were defined accordingly. Some other studies ${ }^{13,14}$ examined the behavior of polymer blend experimentally.

In order to predict the rheological properties of low filled systems, Havet and Isayev applied a thermodynamic approach. ${ }^{15,16}$ As they concluded, the variation of rheological properties with particle volume fraction is because of the change in the relaxation time and the modulus of adsorbed polymer. Sarvestani assumed free and adsorbed polymer chains phase in the filled systems and estimated reptation parameters for these chain types statistically ${ }^{17}$ and thermodynamically ${ }^{18,19}$ without considering any interaction between different phases. For the highly filled systems, filler-filler interaction is considered as the controlling parameters of rheological properties. $^{20,21}$ Song and Zheng applied two-phase model to predict linear viscoelastic of reinforced rubber ${ }^{22}$ and filled plastic melt. ${ }^{23}$ In these studies, filler and bulky polymer phases are considered responsible for the properties of filled systems. Also, Sobhabi et al. ${ }^{24}$ employed Leonov model as continuum ones to predict the viscoelastic behavior of filled polymer melts. They assumed the total stress in the filled systems to be the sum of stress in polymer matrix and filler network.

In this study, the network theory is described for the highly filled systems. Five types of sub-chains are considered, and breakage and regeneration factors are estimated for each sub-chain. In the proposed model, the relaxation modulus as the viscoelastic term is studied for Small Step Strain Test. With regard to the material variables, the effect of particle size and concentration on viscoelastic behavior is predicted. Regeneration and breakup factor as well as breakup ratio are the model constants and their effect on the viscoelastic behavior is investigated. Regarding the model results, the relaxation modulus increases with reduction in particle size and increase in the particle concentration each time. Breakage and regeneration factor and breakup ratio can alter the terminal zone and final state of Small Step Strain Test. We also performed frequency sweep test on polystyrene filled with nano-silica for different particle size and concentration values, along with a general comparison between the experimental and model results.

\section{Model description}

For this study, in order to acquire a suitable model, we consider some assumptions as follows:

- Particles are dispersed randomly in the system.

- The dimension of the single particle is considered in the order of the gyration radius of the polymer chain, i.e. particles are in nano-scale order.

Moreover, a pattern for the behavior and the structure of the polymer chains and particles is required. This pattern can define the elements and their arrangements in the system. Vicinity of polymer chains to the particles provides a chance for the polymer chains to create a junction with particle surface. Particle-polymer and polymer-polymer junctions divide a polymer chain to the different types of polymer sub-chains, where a sub-chain (network segment) is a portion of the chain that ends to the junction or free end, and contains some segments. Two different junctions and a free end position provide different types of sub-chain. The proposed structure of polymer and particle for the highly filled systems is displayed in Figure 1.

In this figure, the particle-polymer and polymerpolymer junctions are indexed with $\mathrm{A}$ and $\mathrm{B}$, respectively. Compared to the other sub-chains, a sub-chain that approaches to the particle surface has different behaviors and properties, which results in different characteristics for the filled systems. On the other hand, filler-polymer interaction leads to adsorbed sub-chains on the surface of the particles. ${ }^{25}$ These sub-chains have a junction with filler surface and are labeled with numbers 2 and 4 in Figure 1. In the highly filled systems, since the particles are very close and polymer chain is capable of embracing some particles, a number of sub-chains connect particles to each other. These type of sub-chains lead to the indirect filler-filler interaction. ${ }^{26,27}$ The sub-chain that is labeled with number 1 has two junctions with the surface of two different particles and these sub-chains are the interaction reagent of fillers with each other. However, some segments such as segments 3 and 5 are not affected by the fillers surface.

The filler-polymer interaction and hydrodynamic motion of the particles in the media are the key parameters controlling the properties of the highly filled systems. In the presented pattern for these systems, sub-chains types 2 and 4 are responsible for the fillerpolymer interaction, and sub-chains such as type 1 are responsible for the filler-filler interaction. Hydrodynamic motion of the particles is a function of particles' structure and the viscosity of the surrounding media. In this regard, the behavior of sub-chains types 3 and 5 determines the viscosity of the free media. 


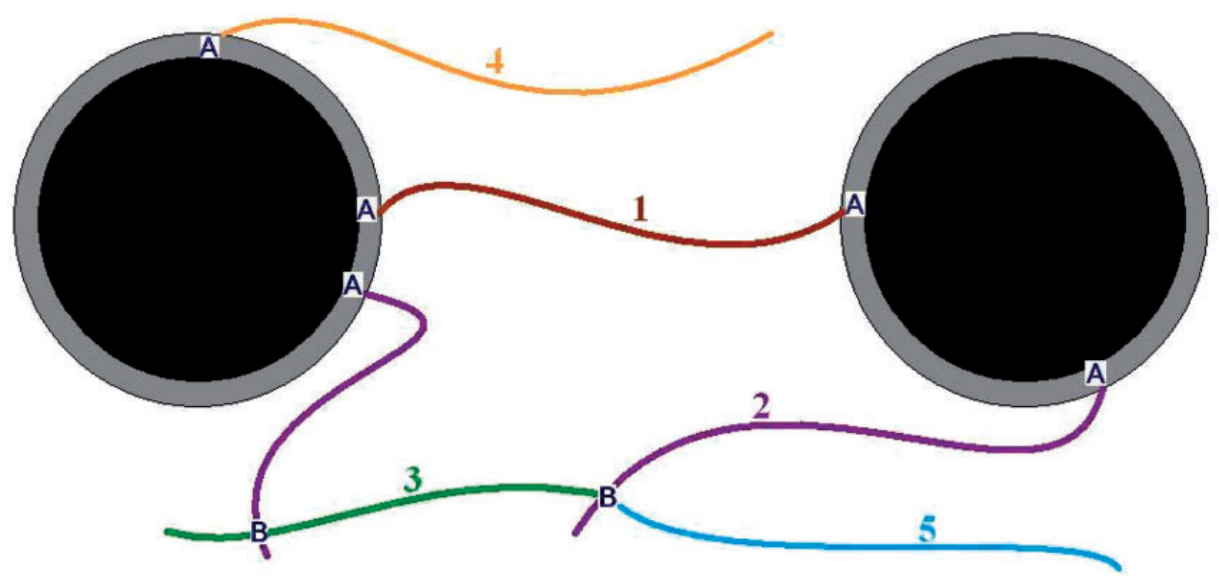

Figure I. Proposed structure for the highly filled systems.

\section{Distribution function}

In order to derive the stress-strain-time relation, Yamamoto used the rubber-like network model, where the junctions break up and reform continually. He also took into account the statistical mechanical consideration of equilibrium state. This relation is as follow

$$
\begin{aligned}
\tau= & \sigma-P I=\sum_{i} \iiint(h h)\left(\frac{1}{r} \frac{\mathrm{d} \varphi(r, N, t)}{d r}\right)_{r=|h|} \\
& \times F(h, N, t) d h
\end{aligned}
$$

In this equation, $\sigma$ is the total stress of the system, $\varphi$ represents the energy of the sub-chains, $N$ is the number of segment in the sub-chains, $h$ denotes the end-to-end vector of the sub-chains, $F$ is the chain distribution function, and $(h h)$ is a tensor. Yamamoto determined the differential for the chain distribution function as follow

$$
\begin{aligned}
\frac{\partial F(h, N, t)}{\partial t}= & -\frac{d e}{d t} e^{1} h \nabla F(h, N, t)-\frac{1}{|e|} \frac{\mathrm{d}|e|}{d t} F(h, N, t) \\
& +P(h, N, t)-L(h, N, t)
\end{aligned}
$$

In this equation, $\mathrm{e}, \mathrm{P}$ and $\mathrm{L}$ represent the deformation tensor, the regeneration rate of junctions, and the breakup rate of the junctions, respectively.

In the highly filled systems, the total stress of the system is considered as the summation of the stress exerted by all types of sub-chains multiplied by its volume fraction

$$
\tau=\sum_{i} \tau_{i} v_{i}
$$

Determination of the regeneration and breakup rate of the junction for each sub-chain is the major concern of this study. It is assumed that the junctions are reversible so the regeneration and breakup could be considered as stochastic reversible process, thus the regeneration rate is

$$
\begin{aligned}
& P_{1}=\alpha_{A} F_{4} \\
& P_{2}=\alpha_{B} F_{4}+\alpha_{A} F_{5} \\
& P_{3}=\alpha_{B} F_{5} \\
& P_{4}=2 \beta_{A} F_{1}+\beta_{B} F_{2} \\
& p_{5}=\beta_{A} F_{2}+2 \beta_{B} F_{3}
\end{aligned}
$$

In these relations, $\alpha_{A}$ and $\alpha_{B}$ are the generation coefficients of the polymer-filler and polymerpolymer junctions, respectively. The breakup coefficients of the polymer-particle and polymer-polymer junctions are represented with $\beta_{\mathrm{A}}$ and $\beta_{\mathrm{B}}$, respectively. The breakup rate can be presented the same as the regeneration rate

$$
\begin{aligned}
& L_{1}=2 \beta_{A} F_{1} \\
& L_{2}=\beta_{A} F_{2}+\beta_{B} F_{2} \\
& L_{3}=2 \beta_{B} F_{3} \\
& L_{4}=\alpha_{A} F_{4}+\alpha_{B} F_{4} \\
& L_{5}=\alpha_{A} F_{5}+\alpha_{B} F_{5}
\end{aligned}
$$

Introducing the regeneration and breakup for each sub-chain, defined in equations (4) and (5), into equation (2), provides us the equations for distribution function for these sub-chains. These equations are dependent, so the distribution function of each sub-chain can be achieved by solving these equations simultaneously. The final model that can describe the behavior of the highly filled model can be presented 
as follow

$$
\begin{aligned}
\frac{\partial F_{1}(h, N, t)}{\partial t}= & -\frac{d e}{d t} e^{-1} h \nabla F_{1}(h, N, t) \\
& -\frac{1}{|e|} \frac{\mathrm{d}|e|}{d t} F_{1}(h, N, t)+\alpha_{A} F_{4}(h, N, t) \\
& -2 \beta_{A} F_{1}(h, N, t) \\
\frac{\partial F_{2}(h, N, t)}{\partial t}= & -\frac{d e}{d t} e^{-1} h \nabla F_{2}(h, N, t)-\frac{1}{|e|} \frac{\mathrm{d}|e|}{d t} F_{2}(h, N, t) \\
& +\alpha_{B} F_{4}(h, N, t)+\alpha_{A} F_{5}(h, N, t) \\
& -\beta_{A} F_{2}(h, N, t)-\beta_{B} F_{2}(h, N, t) \\
\frac{\partial F_{3}(h, N, t)}{\partial t}= & -\frac{d e}{d t} e^{-1} h \nabla F_{3}(h, N, t)-\frac{1}{|e|} \frac{\mathrm{d}|e|}{d t} F_{3}(h, N, t) \\
& +\alpha_{B} F_{5}(h, N, t)-2 \beta_{B} F_{3}(h, N, t) \\
\frac{\partial F_{4}(h, N, t)}{\partial t}= & -\frac{d e}{d t} e^{-1} h \nabla F_{4}(h, N, t)-\frac{1}{|e|} \frac{\mathrm{d}|e|}{d t} F_{4}(h, N, t) \\
& +2 \beta_{A} F_{1}(h, N, t)+\beta_{B} F_{2}(h, N, t) \\
& -\alpha_{A} F_{4}(h, N, t)-\alpha_{B} F_{4}(h, N, t) \\
\frac{\partial F_{5}(h, N, t)}{\partial t}= & -\frac{d e}{d t} e^{-1} h \nabla F_{5}(h, N, t)-\frac{1}{|e|} \frac{\mathrm{d}|e|}{d t} F_{5}(h, N, t) \\
& +\beta_{A} F_{5}(h, N, t)+2 \beta_{B} F_{5}(h, N, t) \\
& -\alpha_{A} F_{5}(h, N, t)-\alpha_{B} F_{5}(h, N, t) .
\end{aligned}
$$

\section{Small Step Strain Test}

Small Step Strain Test is a basic experiment for studying linear viscoelastic response of the polymeric systems. ${ }^{6}$ Employing the linear viscoelastic relations, one can estimate the rheological properties such as elastic and loss modulus, as well as dynamic viscosity, using the relaxation modulus. In the relaxation test, the deformation is constant and is not a function of time, so the resultant of equation (6) for this type of test is presented in equation (7) which can provide us the distribution function of each sub-chain in the relaxation test.

$$
\begin{aligned}
\frac{\partial F_{1}(h, N, t)}{\partial t}= & \alpha_{A} F_{4}(h, N, t)-2 \beta_{A} F_{1}(h, N, t) \\
\frac{\partial F_{2}(h, N, t)}{\partial t}= & \alpha_{B} F_{4}(h, N, t)+\alpha_{A} F_{5}(h, N, t) \\
& -\beta_{A} F_{2}(h, N, t)-\beta_{B} F_{2}(h, N, t) \\
\frac{\partial F_{3}(h, N, t)}{\partial t}= & \alpha_{B} F_{5}(h, N, t)-2 \beta_{B} F_{3}(h, N, t) \\
\frac{\partial F_{4}(h, N, t)}{\partial t}= & 2 \beta_{A} F_{1}(h, N, t)+\beta_{B} F_{2}(h, N, t) \\
& -\alpha_{A} F_{4}(h, N, t)-\alpha_{B} F_{4}(h, N, t) \\
\frac{\partial F_{5}(h, N, t)}{\partial t}= & \beta_{A} F_{2}(h, N, t)+2 \beta_{B} F_{3}(h, N, t) \\
& -\alpha_{A} F_{5}(h, N, t)-\alpha_{B} F_{5}(h, N, t)
\end{aligned}
$$

Regeneration and breakup coefficient for the particlepolymer junction and polymer-polymer junction are the molecular parameters that determine and control the properties of the filled systems. In the linear region, the distribution function of sub-chains is independent of its end-to-end distance. This assumption is considered to solve the tension equation for the highly filled system. The relaxation modulus resulted from this assumption along with equations (1) and (3) is

$$
\begin{aligned}
G(h, N, t) & =\frac{\tau}{\gamma_{0}}=\frac{\sum_{i} v_{i} \tau_{i}}{\gamma_{0}} \\
& =\frac{1}{\gamma_{0}} \sum_{j} \iiint(h h)\left(\frac{1}{r} \frac{\mathrm{d} \phi(r, N, t)}{d r}\right)_{r=|h|} d h \times q=\varepsilon q \\
q & =\sum_{i} v_{i} F_{i}(h, N, t) .
\end{aligned}
$$

In this equation, $\varepsilon$ is constant, so $\mathrm{q}$ represents the relaxation modulus. It is clear that, besides the molecular parameters, volume fraction of each sub-chain alters the relaxation modulus and other rheological properties of the filled systems. The volume fractions of each sub-chain change with the particle size and volume fraction. Higher particle volume fraction provides higher surface area for the polymer adsorption and higher fractions for the sub-chains which are labeled as 2 and 4. Furthermore, higher particle volume fraction leads to lower inter-particle distance, which means higher fraction for the sub-chain labeled with 1 . Smaller particles are more numerous and have higher surface compared to larger ones, and therefore these particles behave in the same manner as the higher volume fraction of larger particles. The above equations are solved in MATLAB.

The initial condition of the system is important for solving equation (7). The initial condition or static properties as well as the properties of components depend on the morphology of the system and the interactions of the components. Also, the morphology of the systems is determined with their mixing conditions ${ }^{28}$ and their thermodynamics. ${ }^{29,30}$

\section{Experimental}

\section{Materials}

Polystyrene grade 336 provided by EN CUAN Industrial, with a density of $1.05 \mathrm{~g} / \mathrm{cm}^{3}$ (ISO 1183) and a melt flow index of $12(\mathrm{~g} / 10 \mathrm{~min})$ was used as a matrix. Non-porous Silica Aerosil 200, 90, OX50 (supplied by Degussa Chemical) was used for the preparation of the nanocomposites under investigation. All silica particles are hydrophilic with surface area of 200,90 , and $50 \mathrm{~m}^{2} / \mathrm{g}$, and their average 
primary particle sizes are 12,20 , and $40 \mathrm{~nm}$, respectively.

\section{Sample preparation}

Mixing silica powder with the PS matrix was performed in toluene according to the following procedure. First Silica powder was sonicated in toluene for $30 \mathrm{~min}$, and then PS was added to the suspension under magnetic stirring for $1 \mathrm{~h}$. The mixture was subjected to high shear mechanical stirring at room temperature for $6 \mathrm{~h}$. The resulting solution was cast on a Teflon sheet followed by drying for six days and vacuum drying at $60^{\circ} \mathrm{C}$ for one day.

The PS-SiO2 melt samples were prepared with internal mixer at a rotational speed of $50 \mathrm{r} / \mathrm{min}$ for $10 \mathrm{~min}$ at $200^{\circ} \mathrm{C}$. The collected molten materials were compression-molded into $1-\mathrm{mm}$ thick and $25-\mathrm{mm}$ diameter plates by hot pressing under 100 bar for $20 \mathrm{~s}$ at $200^{\circ} \mathrm{C}$.

\section{Rheological measurements}

Dynamic measurements were performed by a Stress controlled rotary shear rheometer (Paar-Physica UDS 200) using a plate-plate geometry. For samples having a high viscosity or permanent elasticity, plate-plate geometry is more suitable than cone and plate geometry due to ease of gap control in this geometry. The frequency sweep rheometry was performed in the frequency range of $0.01-500 \mathrm{~Hz}$, at $1 \%$ deformation and a temperature of $200^{\circ} \mathrm{C}$.

\section{Model study}

There are two parameters that control the properties of composites: the behavior of each sub-chain and their volume fractions. Moreover, the properties and behavior of the each sub-chain vary with the physics of the system and the interaction between the components. As the available surface area and volume fraction of the particle cannot alter the behavior of the sub-chains, their volume fraction will change with particle surface area and concentration. The major challenge in this study is the estimation of volume fraction of each sub-chain. In the highly filled systems, available surface area of particles are high enough to absorb all the polymers' segments and the total modulus of the system are high compared to the free polymers. Thus the assumption that considers the effects of segment numbers 4 and 5 on the total properties of the system negligible seems reliable. The volume fraction of the sub-chains that are labeled with number 2 can be estimated from the total volume fraction of the adsorbed polymers, i.e. the volume faction of the adsorbed polymers can be estimated from the volume of the chain adsorbed layer on the particle surface as follow

$$
\varphi_{a}=s v \rho z
$$

where $s$ is the surface area of filler particle that is available for the polymer chains to be adsorbed, $v$ is the volume fraction of filler in the composites, $\rho$ is the density of particle structure, and $z$ is the thickness of adsorbed polymer layer. The mixing quality of composites and the physical properties of particle surface would change the available surface area of the particles. Particles' structures are puffy and their density is lower than the single particle. Better interaction of the polymer chains with particles and lower interaction of the particles with each other would help particle structure destruction with mixing and reaching the single particles state in the composites instead of the aggregates. These conditions lead to higher density of the particle structure and the resulting composite. The adsorption of polymer chains on the particle surface is because of the hydrogen bonding, and the strength of the hydrogen bonding changes with the polarity of these two components. The amount of adsorbed polymer increases with the adsorbed energy because of the hydrogen bonding and other interfacial interaction energy. ${ }^{31}$

For the highly filled systems, the inter-particle distance is small which decreases with the increase in particle concentration and surface area. Lower interparticle distance leads to higher amount of segment number 1 , so the amount of this type is an inverse function of this parameter. From the following equation, the inter-particle distance can be estimated from the particle volume fraction and surface area

$$
\delta_{\text {agg }}=\frac{6000}{\rho s}\left(K v^{-1 / 3} \beta^{-1 / 3}-1\right) \beta^{1.43}
$$

In this equation, $K$ is constant and for spherical filler is equal to $0.806 . \beta$ is the ratio of effective to real volume fraction which is a function of physical properties of composite's components. For compatible components, $\beta$ is approximately 1 and for noncompatible components it is less than 1 , therefore by referring to an aggregate's dimension and introducing the mixing quality of the system, one can estimate this parameter.

In order to study the behavior of highly filled systems, some constants in equation (7) are required to be clarified. Some of these constants (called as model parameters) are based on the behavior of free polymer subchains and adsorbed polymer at particle surface, such as the breakage factor of the free sub-chains. Other types of the constants (called as material constants) are functions of polymer and particle physical 
properties, which are used to estimate the volume fraction of each sub-chain, such as particle density and adsorbed polymer layer. In Table 1, these constants are presented.

In addition to the constants, defined and presented in the previous equations, the breakup ratio is defined as ratio of the breakup coefficient of the polymerparticle to the polymer-polymer junctions. This parameter can be a criterion for the ratio of polymer interaction with polymer-polymer interaction with the particle surface. In the known filled system, this parameter can be altered using surface modification of particles with other groups instead of the primary groups.

In the following sections, the effect of the defined constants and variables is studied. The final goal of modeling is to predict the properties of the known system or design a system with dictated properties for the known process or application. These constants and variables are tools to reach the final goals of the modeling. The effect of the particle concentration and surface area and the constants of the model, used and defined in equations (4) and (5), are the parameters investigated here.

\section{Particle volume fraction}

Configuration of chains in the space changes at the solid or crystalline surfaces. With this change, which is related to the adsorption energy, entropy of chains decreases. ${ }^{32}$ Chains which are not directly connected to the filler surface but are next to the directly adsorbed chains also have lower entropy compared to the free chains. Therefore, these overall adsorbed chains form

Table I. Constants determining the behavior of highly filled systems.

\begin{tabular}{ll}
\hline Constants & $\begin{array}{l}\text { The constants } \\
\text { symbol }\end{array}$ \\
\hline $\begin{array}{l}\text { Polymer-polymer regeneration } \\
\text { junction coefficient }\end{array}$ & $\alpha_{\mathrm{B}}$ \\
$\begin{array}{l}\text { Polymer-particle regeneration } \\
\text { junction coefficient }\end{array}$ & $\alpha_{\mathrm{A}}$ \\
$\begin{array}{l}\text { Polymer-particle breakup junc- } \\
\quad \text { tion coefficient }\end{array}$ & $\beta_{\mathrm{A}}$ \\
$\begin{array}{l}\text { Polymer-polymer breakup junc- } \\
\text { tion coefficient }\end{array}$ & $\beta_{\mathrm{B}}$ \\
$\begin{array}{l}\text { Breakup ratio } \\
\text { Adsorbed polymer layer } \\
\text { thickness }\end{array}$ & $\lambda=\beta_{\mathrm{A}} / \beta_{\mathrm{B}}$ \\
$\begin{array}{l}\text { Available surface area of particles } \\
\text { Particle structure density }\end{array}$ & $\mathrm{S}$ \\
\hline
\end{tabular}

a shell near the particles. The thickness of this shell and its modulus are the parameters that control the properties of the composite. The thickness and modulus of the adsorbed layer are functions of directly adsorbed polymer chains' entropy or adsorption energy.

With an increase in the filler volume fraction, available surface area for adsorption of polymer chain increases. Adsorbed polymer chains have different properties compared to the free chains, ${ }^{10}$ so with the increase in the volume fraction of filler composite, the rheological properties could change. With this point of view, the increase in particle volume fraction leads to increase in the volume fraction of sub-chains type 2 and 4. Forasmuch as each type of the existence subchains has different properties, the change in their volume fraction has an effect on the overall properties of the system.

On the other hand, the number of particles in a unit volume increases with an increase in particle concentration, so inter-particle distances decreases. This statement could be confirmed with equation (10). The reduction in inter-particle distance leads to increase in the volume fraction of sub-chains type 1 . The properties of the highly filled composite are related to filler concentration because of the relation of adsorbed polymer types 1 and 2 volume fraction to this parameter.

In Figure 2, the modulus of the system is estimated at the different particle volume fraction. This estimation is performed by solving equation (7), and the result is inserted into equation (8) and the outcome is shown in this figure in logarithmic scale. The value of the constants that are used to study the effect of the particle volume fraction is presented in Table 2 .

The presented constants in this table may have some effects on the properties of the system, and therefore

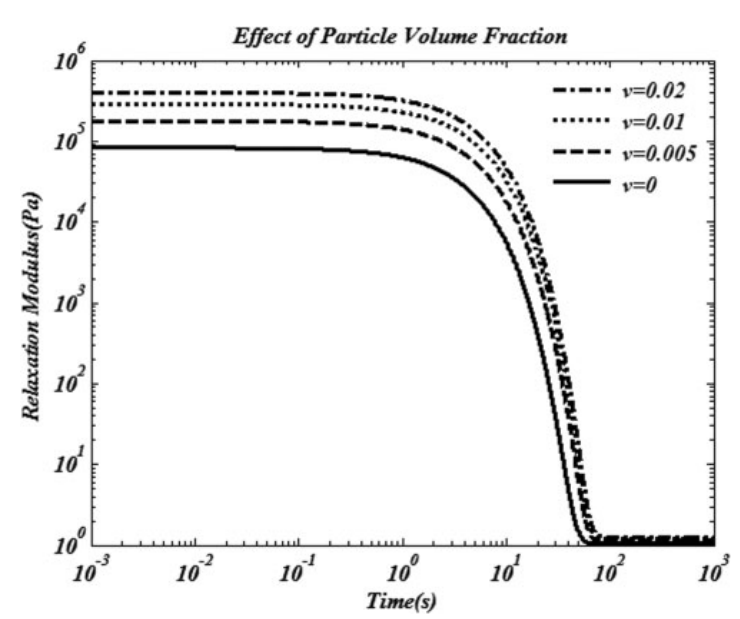

Figure 2. Modulus of the system at different particle volume fractions. 
Table 2. The values of the constant that are used to study the effect of the particle concentration.

\begin{tabular}{llll}
\hline Constant & Symbol & Value & Unit \\
\hline Available surface area of particle & $\mathrm{S}$ & 100 & $\mathrm{~m}^{2} / \mathrm{g}$ \\
$\begin{array}{l}\text { Adsorbed polymer layer } \\
\text { thickness }\end{array}$ & $\mathrm{Z}$ & 22 & $\mathrm{~nm}$ \\
$\begin{array}{l}\text { Particle structure density } \\
\begin{array}{l}\text { Polymer-particle regeneration } \\
\text { junction coefficient }\end{array}\end{array}$ & $\mathrm{P}$ & 2.1 & $\mathrm{~g} / \mathrm{m}^{3}$ \\
$\begin{array}{l}\text { Polymer-polymer regeneration } \\
\text { junction coefficient }\end{array}$ & $\alpha_{\mathrm{B}}$ & $10^{-6}$ & \\
$\begin{array}{l}\text { Polymer-particle breakup junc- } \\
\text { tion coefficient }\end{array}$ & $\beta_{\mathrm{A}}$ & $10^{-5}$ & \\
\begin{tabular}{l} 
Breakup ratio \\
\hline
\end{tabular} & $\Lambda=\beta_{\mathrm{A}} / \beta_{\mathrm{B}}$ & 0.1 & \\
\hline
\end{tabular}

these constants can be considered as the studying parameters. Available surface area of the particle and breakup and regeneration coefficient of the junctions are investigated in the following sections. The materials in the case study of this study, which define the material constants, particle surface area, adsorbed layer thickness and particle structure density, are polystyrene and nano-silica. And for the model constants such as regeneration factor, the non-base value is considered.

At each time, increase in the modulus with the increase in the volume fraction of the particle is predicted. The result of this prediction at short and long times is interesting; the difference between the modulus at different volume fractions at the short times is higher than at the long times, which indicates that the filled systems relax to the same state at a long time case and existence of the particles changes the initial state of the systems.

This study is performed on the polymer melt that is filled with a high fraction of nano-particles. In the melt rheology of polymers, the plateau region is observed and this study can predict this region as well. For the neat polymer, the modulus of this region is a function of the molecular weight of the polymer chains, i.e. the density of junctions. Existence of the particles provides a new chance for the polymer chains to create a junction with particle surface. This junction determines other types of sub-chain. The density of every type of junction or the volume fraction of the sub-chains determines the modulus of the plateau region and the density of each type of the junction can change with the particle size and concentration. Increase in the modulus of this region with the volume fraction of added particles is predicted. Other region in the melt rheology is terminal. In this region, the modulus of the system starts to decrease. This model defines the terminal region for the highly filled systems as well.

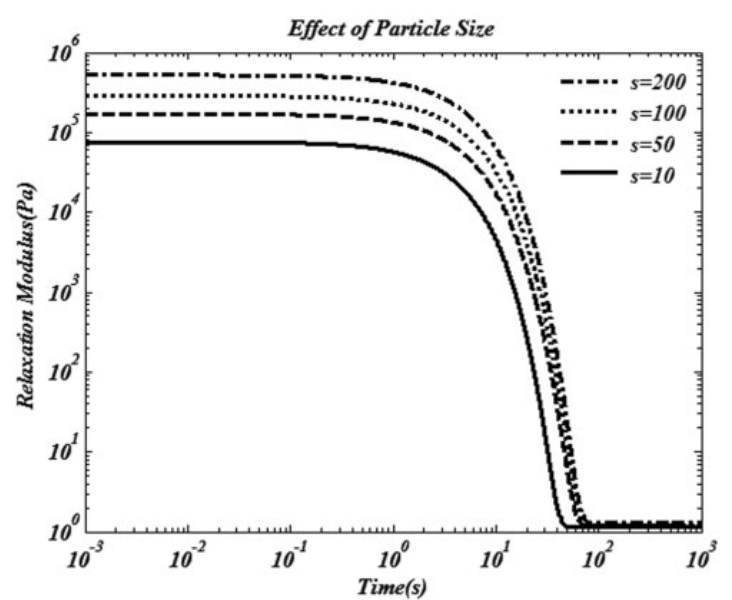

Figure 3. Effect of the surface area or size of the particles.

\section{Particle surface area}

The effect of filler particle size on the composite properties is similar to the filler particle concentration. With the reduction in particle size, the number of particles in a given volume increases and the available surface of filler for adsorption of chains increases as well.

Filler-filler interactions could be divided into direct and indirect interactions. At higher particle concentration, adsorbed layers around particles can overlap and form a 3D structure of fillers in the filled system. In this state, stresses that are exerted onto the system transfer between fillers through the adsorbed layer, so fillers can interact on each other indirectly. In the direct fillerfiller interaction, fillers connect to each other directly and create aggregate, chains and agglomerates. ${ }^{20}$ There is no polymer chain inside the aggregate structures because these structures are dense and they are not destructed during the mixing process.

Like the increase in the volume fraction, increase in the surface area of particle increases the probability of creating sub-chains type 1,2 and 4 . The volume fraction of sub-chain type 1 , as the most important parameter that control the properties of the highly filled system, increases with the decrease in the inter-particle distance. Inter-particle distance decreases with the increase in particle number in the system, so particle surface area can have an effect on the properties of highly filled systems by changing the volume fraction of the sub-chain type 1 .

In Figure 3, the effect of particle size or surface area is investigated. The volume fraction of the particle is considered 0.2 and the other constants are the same as the constants used for studying the particle volume fraction shown in Table 2.

This study predicts the increase in the investigated parameter, which presents the modulus of the highly filled systems, with increase in the surface area of the particles. 
The difference between this parameter at initial times is higher than the long times which means the modulus of these systems decay to the same state at longer times and the usage of the smaller particles just changes in the initial state of the systems.

\section{Studying the breakage factor}

Breakage factor is a parameter that determines the resistance to the breakage of the segments' junctions to the applied forces or Brownian movements of the polymer molecules. Breakage in the junctions leads to the decrease in the modulus of the systems, so the result of lower breakage factors is higher resistance to the decay in the applied stress. This parameter could be changed with the temperature and pressure of the test as well as the amplitude of the applied stress or strain. At higher temperature or lower pressure, polymeric systems start to decay the applied stress at shorter times, so these variables could change the breakage factor. Higher applied stress or strain provides a different condition for the system. Linear and non-linear properties are the product of amplitude of the applied stress or strain, and change in the behavior of the polymeric systems at different amplitude of the stress or strain could be due to the variation in the breakage factor with this variable accordingly.

This behavior is studied for the highly filled systems. For these systems, two types of breakage is possible; breakage of the junction of the polymers' segments with each other and the junction of the polymer segments with the particles' surfaces. Because of the polar group on the surface of the particles, the interaction of the polymer segments with particles is higher than the polymer-polymer interaction, and therefore the breakage factor for the polymer-polymer junction is higher than the polymer-particle junction. The effect of breakup coefficient on the relaxation modulus is shown in Figure 4.

It is found that with increase in the breakage factor, the modulus of the system starts to decrease at shorter times. In this type of tests, a time constant could be defined as the times that the system is needed to relax to the certain fraction of the applied external stress. It is clear from Figure 4 that these constant times would decrease with the increase in the breakage factor.

\section{Regeneration factor}

In this study, the regeneration factor is considered as the tendency of the junction to rebuild after the breakage or generation of a new junction in the system with change in its equilibrium condition. To break the junction in the system, some energy is needed to overcome the breakup. This energy provides the driving force to

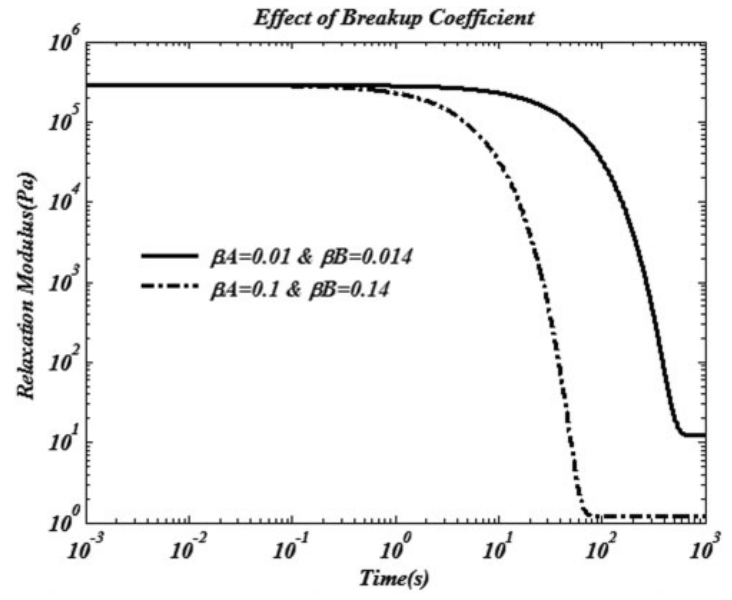

Figure 4. Study of the breakup coefficient.

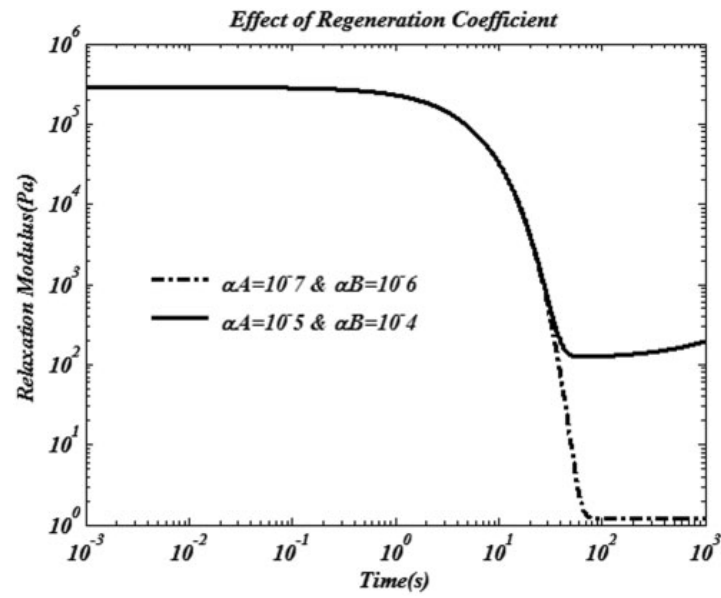

Figure 5. Study of the regeneration coefficient of the junctions.

regenerate this junction. On the other hand, the movement of the polymer chains in the polymer network provides a chance for sub-chains to create a new junction. Those possibilities to generate a junction in the systems after change in the equilibrium condition states determine the regeneration factor. Temperature, pressure, frequency and amplitude of the applied forces and physical interaction of the polymer and particle in the system can have an effect on this parameter.

Higher regeneration coefficient means lower loss in the applied stress in the system. For the dynamic tests, regeneration phenomena can predict thixotropic and rheopexy behavior for the polymeric and other systems with network structure. The effect of the regeneration coefficient on the relaxation modulus is shown in Figure 5.

The initial and final state of the system, the time when a modulus of the system starts to decrease, and the zone of transition from the initial to the final state are properties of the system that are a function of the 
breakage and regeneration coefficient of each type of the junction. For the same initial state of the system, it is shown in Figure 5 that higher regeneration of the junctions leads to the higher final state.

\section{Breakup ratio}

The breakup ratio is considered as the ratio of the breakup coefficient of polymer-particle junction to the polymer-polymer junction. The quantity of this constant determines the tendency of the polymer sub-chains to create a junction with other polymer subchains or the surface of the particles. Higher amount of

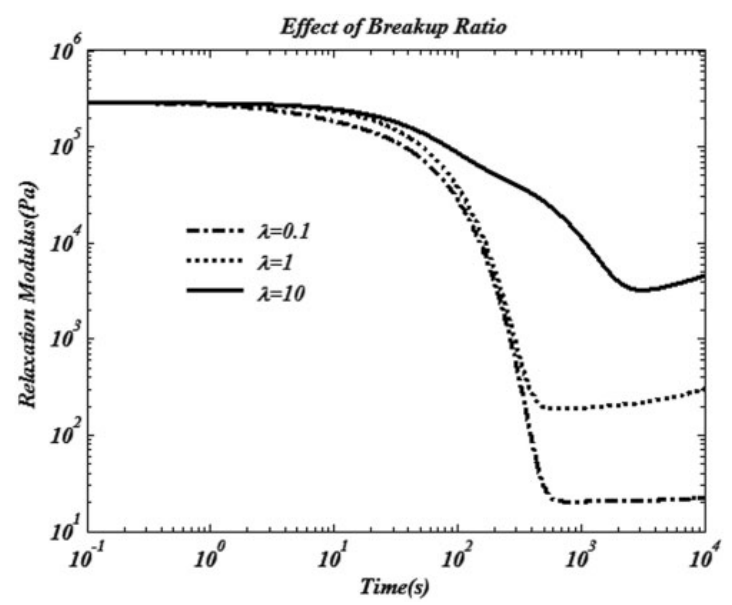

Figure 6. Study of the effect of the breakup ratio. this constant expresses the lower tendency of the polymer to attach to the particle surface compared to entangle with polymer network. In the real systems, this constant could be controlled with change in the surface properties particles and the nature of the polymer backbone. The constant is studied for the values of $0.1,1$, and 10, and the results are presented in Figure 6.

Regarding the data shown in Figure 6, it is clear that lower breakup ratio leads to the terminal zone where the studied property starts to decrease at higher times. Polymer chains can anchor on the particle surface while interacting with the occupant group on the particles' surface and create a junction, so the strength of anchoring on the particle surface can change the breakup ratio and this strength could be attributed to the physics of the particle surface. In the practical study, breakup ratio can change with surface modification. When the occupant groups were replaced with inert group that have lower interaction with polymer chains, the breakup ratio increases and higher interaction would decrease this parameter.

\section{Comparison with experimental results}

The common test used for confirming the model prediction is frequency sweep, because this test can separate viscose and elastic response of materials, the requirement which is needed for our case. Fast Fourier transform is also employed to convert the data in time domain estimated from equation (7) into frequency domain. The relation between time domain data and frequency

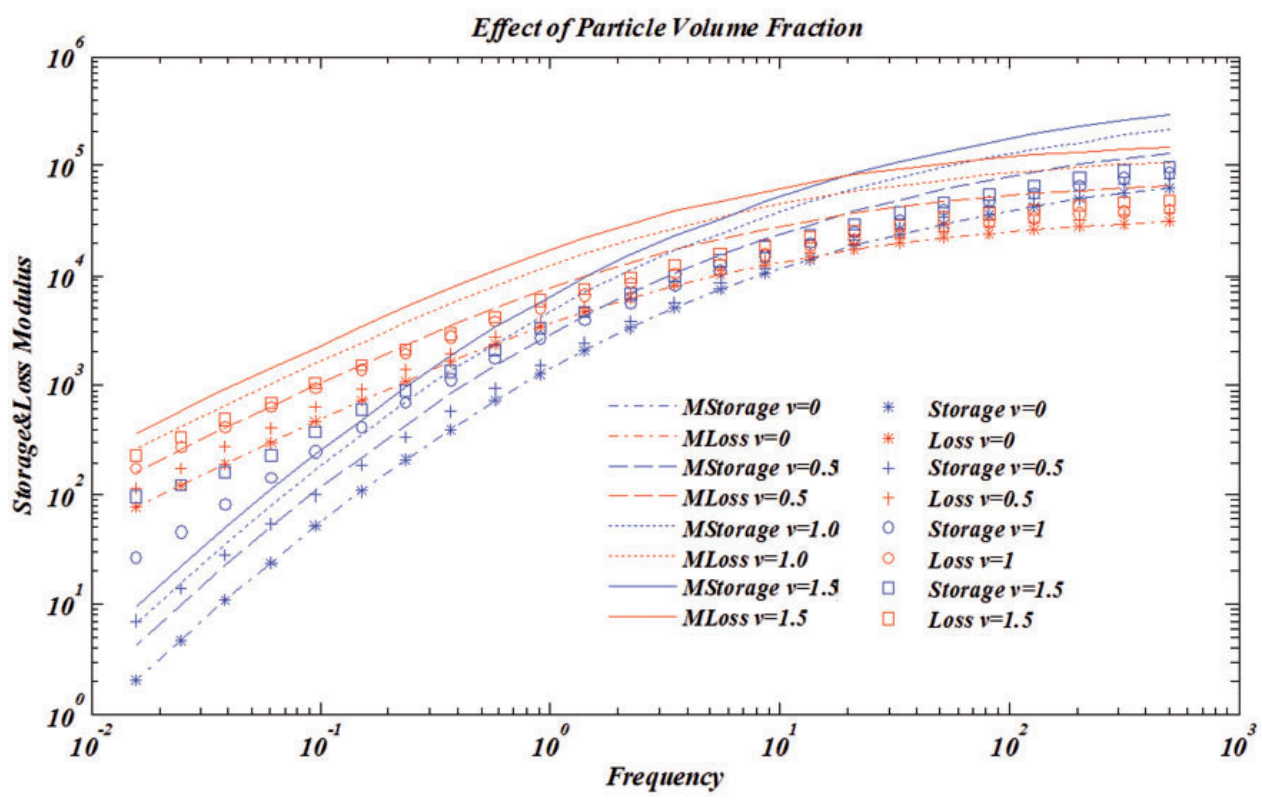

Figure 7. Effect of particle concentration on the storage and loss modulus: experimental and model comparison. 
domain is as follows

$$
\begin{aligned}
& G^{\prime}(\omega)=\omega \int_{0}^{\infty} G(t) \sin (\omega t) d t \\
& G^{\prime \prime}(\omega)=\omega \int_{0}^{\infty} G(t) \cos (\omega t) d t
\end{aligned}
$$

In this equation, $G^{\prime}$ is elastic modulus and $G^{\prime \prime}$ is loss modulus. Experimental data showed increase in the

Table 3. Constants used in the prediction of experimental data.

\begin{tabular}{llll}
\hline Constant & Symbol & Value & Unit \\
\hline $\begin{array}{l}\text { Available surface area of } \\
\text { particle for 12, } 20 \text { and } \\
40 \mathrm{~nm}\end{array}$ & $\mathrm{~s}$ & $\begin{array}{r}80,60 \\
\text { and } 37.5\end{array}$ & $\mathrm{~m}^{2} / \mathrm{g}$ \\
$\begin{array}{l}\text { Adsorbed polymer layer } \\
\text { thickness }\end{array}$ & $\mathrm{z}$ & 22 & $\mathrm{~nm}$ \\
$\begin{array}{l}\text { Particle structure density } \\
\begin{array}{l}\text { Polymer-polymer } \\
\text { breakup junction } \\
\text { coefficient }\end{array}\end{array}$ & $\rho$ & 2.1 & $\mathrm{~g} / \mathrm{m}^{3}$ \\
$\begin{array}{l}\text { Breakup ratio } \\
\text { Polymer-polymer regen- } \\
\text { eration junction } \\
\text { coefficient }\end{array}$ & $\beta_{\mathrm{B}}$ & 8 & \\
$\begin{array}{l}\text { Polymer-particle regener- } \\
\text { ation junction } \\
\text { coefficient }\end{array}$ & $\alpha_{\mathrm{A}} / \lambda$ & $10^{-6}$ & \\
\hline
\end{tabular}

storage and loss modulus with respect to the increase in the particle volume fraction, especially at lower frequency values. These observations can be explained according to the increase of particles' total surface area available for polymer chains for adsorption as well as the decrease in the inter-particle distance. Theoretically, higher surface area leads to higher volume fraction for sub-chain types 2 and 4 , and lower inter-particle distance leads to higher volume fraction of sub-chain type 1. In Figure 7, the effect of particle concentration on the loss and storage modulus of composites containing $12 \mathrm{~nm}$ particles along with the prediction results is shown. With regard to this prediction, some constants were employed which are presented in Table 3.

Breakup and regeneration coefficients of a free polymer are estimated by fitting the model with the experimental data of the free polymer. Available surface area, adsorbed layer thickness and density of particle structure are estimated from the density of nanocomposites. ${ }^{33}$ The value of $\lambda$ is determined from the WLF equation at $193^{\circ} \mathrm{C}$, i.e. $7^{\circ} \mathrm{C}$ lower than test temperature, ${ }^{25}$ and the test temperature $\left(200^{\circ} \mathrm{C}\right)$ is considered as the reference temperature.

It is clear from equation (7) that the predicted data are the projection of the free and adsorbed subchain behaviors. Polymeric structure relaxes at higher times, which means the quantity of storage and loss modulus verges to zero at lower frequencies. This behavior is observed for the experimental data of those systems containing lower filler. Regarding the

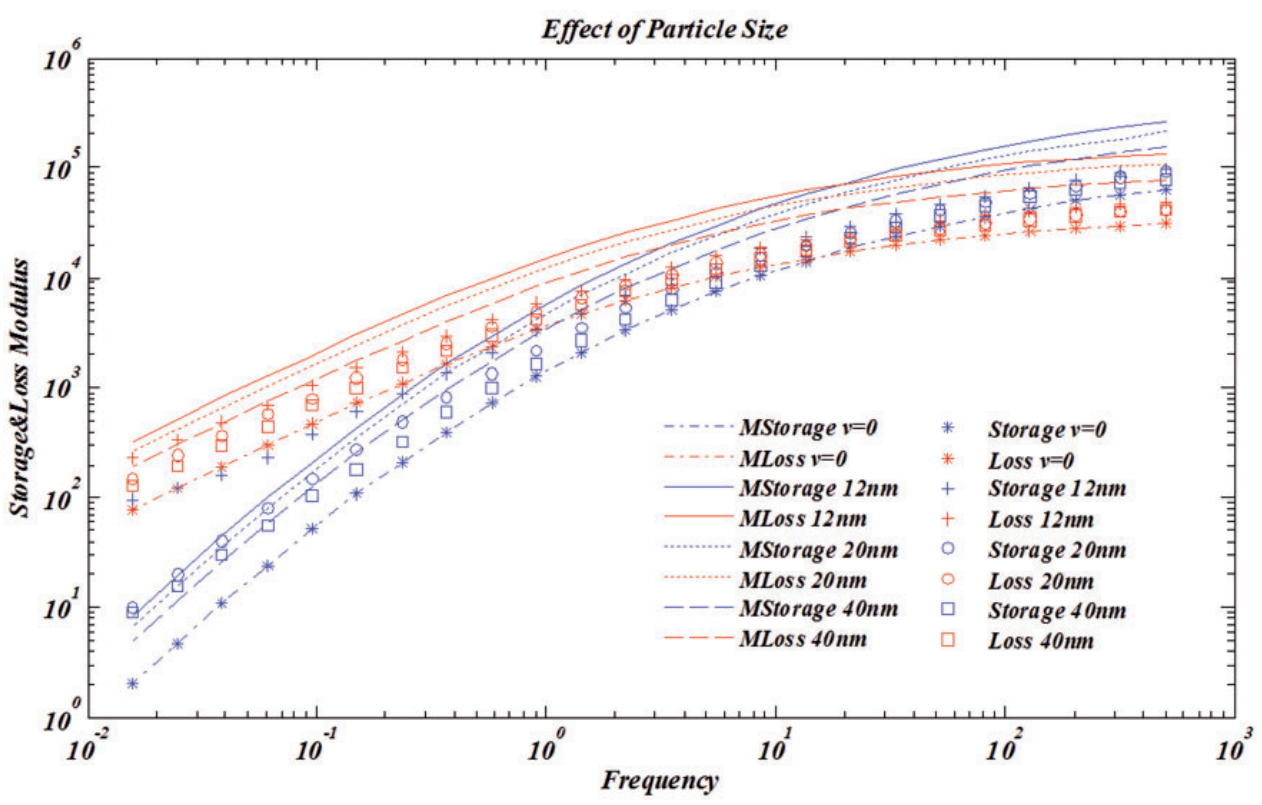

Figure 8. Effect of particle size on the storage and loss modulus at $1.5 \%$ particle volume fraction: experimental and model comparison. 
higher concentration values, the experimental data revealed that the storage modulus value does not tend to zero. This behavior is called yielding. So the difference between experimental and predicted data, i.e. yielding, is not related to the behavior of free and adsorbed polymer sub-chains. Therefore, yielding is the result of the dynamics of particle structure.

In Figure 8, the effect of particle size on the loss and storage modulus of composites containing 12, 20, and $40 \mathrm{~nm}$ nano-silica at $1.5 \%$ particle volume fraction along with the model results is shown. It is clear that experimental loss and storage modulus increase with reduction in particle size because of increase in the available surface area of particle and reduction in inter-particle distance.

We observed the yielding in the data of storage modulus for the systems containing smaller fillers. It can be concluded that the portion of particles' structure dynamics on the system behavior increased with increase in particle fraction and reduction in particle size.

In this study, the behavior of nanocomposites is studied theoretically and experimentally. In the theoretical study, the regeneration and breakup coefficients and the breakup ratio are defined and their effects on the behavior of composites are analyzed. Performing the model analysis, the effects of particle size and concentration are investigated and compared with the experimental results.

\section{Conclusion}

In this research, the behavior of highly filled systems was investigated, and a specific pattern for the molecular structure of these systems was suggested. In this pattern, five types of sub-chain were presented, where some of them belong to the polymer network (type 3). Moreover, in the highly filled systems, particles are close enough to be embraced by a polymer chain which forms a strong indirect network of particles. Sub-chain type 1 is responsible for these sub-chains. In this pattern, one type of end-free sub-chain is defined which is connected from the other end to the particle surface (type 4) or polymer network (type 5). And type 2 is the sub-chain which is connected to particle surface and polymer network. In the filled systems, the attached sub-chains to the particle surface have different properties and provide different properties for the filled system compared to the neat polymers.

Breakup and regeneration factors for a junction are the parameters of Yamamoto model. In the filled system, polymer-particle junction is the exit, so these parameters are defined for that junction. Yamamoto network relation is employed to predict the behavior of every type of sub-chain while defining their regeneration and breakup rate. Yamamoto relations estimate the applied stress to a sub-chain, where the total system at a known deformation and the modulus of the system are determined from the total stress.

The presented model, based on Yamamoto network for highly filled systems, is studied for Small Step Strain Test. In this study, modulus of the system is estimated after applying a certain strain to the system. In this type of test, three regions appeared; initial state, transition zone, and final state. Regeneration factor is studied and it is found that higher regeneration factor leads to higher modulus at the final state. It is concluded that for a system with lower breakage factor, the transition zone occurs at higher times, which means the system has higher strength. Breakup ratio is the parameter that can be defined as breakup coefficient of polymer-particle junction to the polymer-polymer junction. This parameter would be more meaningful when the properties of the interface of polymer and particle change. Surface modification is one of the methods that can alter the properties of interface and breakup ratio. The transition region is seen at initial times when breakup ratio is increased. Particle surface area and volume fraction are material constants that can change the properties of the highly filled system. This model predicts that the initial state of the system increased when these parameter increase.

It is concluded that the properties of a highly filled system can be controlled with the material variables. These variables are related to the properties of system components and interaction of the components with each other. So these parameters could be used in order to predict the properties of a system or help to design a new system with a special characteristic.

At last, we performed a comparison between experimental and predicted data, and we found that for those systems containing higher particle content or smaller particles, particle structure dynamics is outcrop especially in lower frequencies.

\section{Declaration of Conflicting Interests}

The author(s) declared no potential conflicts of interest with respect to the research, authorship, and/or publication of this article.

\section{Funding}

The author(s) received no financial support for the research, authorship, and/or publication of this article.

\section{References}

1. Rouse PE, Jr. A theory of the linear viscoelastic properties of dilute solutions of coiling polymers. J Chem Phys 1953; 21: 1272-1280. 
2. Yamamoto M. The visco-elastic properties of network structure i. General formalism. J Phys Soc Jpn 1956; 11: 413-421.

3. Yamamoto M. The visco-elastic properties of network structure II: structural viscosity. J Phys Soc Jpn 1957; 12: 1148-1158.

4. Yamamoto M. The visco-elastic properties of network structure III. Normal stress effect. J Phys Soc Jpn 1958; 13: $1200-1211$.

5. De Gennes PG. Reptation of a polymer chain in the presence of fixed obstacles. J Chem Phys 1971; 55: 572-579.

6. Doi M and Edwards SF. Dynamics of concentrated polymer systems. Part 1 - Brownian motion in the equilibrium state. J Chem Soc Faraday Transact 2: Mol Chem Phys 1978; 74: 1789-1801.

7. Mewis $\mathrm{J}$ and Wagner NJ. Colloidal suspension rheology. Cambridge University Press, 2012.

8. Advani SG. Flow and Rheology in Polymer Composites Manufacturing. Elsevier, 1994.

9. Fleck F, Froltsov V and Klüppel M. Polymer-filler interphase dynamics and reinforcement of elastomer nanocomposites. Soft Mater 2014; 12: S121-S134.

10. Blum FD and Krisanangkura P. Comparison of differential scanning calorimetry, FTIR, and NMR to measurements of adsorbed polymers. Thermochim Acta 2009; 492: 55-60.

11. Doremus P and Piau JM. Yield stress fluid structural model and transient shear flow behaviour. $J$ NonNewton Fluid Mech 1991; 39: 335-352.

12. Sarvestani AS and Picu CR. Network model for the viscoelastic behavior of polymer nanocomposites. Polymer 2004; 45: 7779-7790.

13. Khademzadeh Yeganeh J, et al. Manipulating the kinetics and mechanism of phase separation in dynamically asymmetric LCST blends by nanoparticles. Phys Chem Chem Phys 2015; 17: 27446-27461.

14. Chaykar AS, Goharpey F and Yeganeh JK. Volume phase transition of electron beam cross-linked thermoresponsive PVME nanogels in the presence and absence of nanoparticles: with a view toward rheology and interactions. $R S C$ Adv 2016; 6: 9693-9708.

15. Havet $\mathrm{G}$ and Isayev AI. A thermodynamic approach to the rheology of highly interactive filler-polymer mixtures. Part I. Theory. Rheol Acta 2001; 40: 570-581.

16. Havet $\mathrm{G}$ and Isayev AI. A thermodynamic approach to the rheology of highly interactive filler-polymer mixtures. Part II. Comparison with polystyrene/nanosilica mixtures. Rheol Acta 2003; 42: 47-55.

17. Sarvestani AS. Modeling the solid-like behavior of entangled polymer nanocomposites at low frequency regimes. Eur Polym J 2008; 44: 263-269.

18. Sarvestani AS, He X and Jabbari E. The role of fillermatrix interaction on viscoelastic response of biomimetic nanocomposite hydrogels. J Nanomater 2008; 2008: 1-9.
19. Sarvestani AS and Jabbari E. Modeling the viscoelastic response of suspension of particles in polymer solution: the effect of polymer-particle interactions. Macromol Theor Simul 2007; 16: 378-385.

20. Kourki H, Mortezaei M and Famili MHN. Filler networking in the highly nanofilled systems. $J$ Thermoplast Compos Mater 2016; 29: 1047-1063.

21. Sarvestani AS. Nonlinear rheology of unentangled polymer melts reinforced with high concentration of rigid nanoparticles. Nanoscale Res Lett 2010; 5: 791-794.

22. Song Y and Zheng Q. Application of two phase model to linear viscoelasticity of reinforced rubbers. Polymer 2011; 52: 593-596.

23. Song $\mathrm{Y}$ and Zheng Q. Application of two phase model to linear dynamic rheology of filled polymer melts. Polymer 2011; 52: 6173-6179.

24. Sobhanie M, Isayev AI and Fan Y. Viscoelastic plastic rheological model for particle filled polymer melts. Rheol Acta 1997; 36: 66-81.

25. Kourki H, et al. Highly nanofilled polystyrene composite: thermal and dynamic behavior. J Elastomer Plast 2016; 48: 404-425.

26. Kourki $\mathrm{H}$ and Famili MHN. Particle sedimentation: Effect of polymer concentration on particle-particle interaction. Powder Technol 2012; 221: 137-143.

27. Sodhani D and Reese S. Finite element based micromechanical modeling of interphase in filler reinforced elastomers. Soft Mater 2014; 12: S152-S161.

28. Capuano $G$, et al. Universal features of the melt elasticity of interacting polymer nanocomposites. Langmuir 2012; 28: 5458-5463.

29. Bartholome C, et al. Viscoelastic properties and morphological characterization of silica/polystyrene nanocomposites synthesized by nitroxide-mediated polymerization. Polymer 2005; 46: 9965-9973.

30. Yeganeh JK, et al. Controlling the kinetics of viscoelastic phase separation through self-assembly of spherical nanoparticles or block copolymers. Soft Matter 2014; 10: 9270-80.

31. Zweistra HJA and Besseling NAM. Mean chain length of adsorbed supramolecular polymers. Phys Rev Lett 2006; 96: 078301-078304.

32. Sarvestani AS and Jabbari E. Modeling and experimental investigation of rheological properties of injectable poly(lactide ethylene oxide fumarate)/hydroxyapatite nanocomposites. Biomacromolecules 2006; 7: 1573-1580.

33. Kourki H, Mortezaei M and Famili MHN. Prediction of the viscoelastic response of filler network in highly nanofilled polymer composites. Journal of Composite Materials 2015; 49: 3799-807. 